ISSUES IN PUBLIC HEALTH

\title{
Human papillomavirus vaccination acceptance and hesitancy in South Africa: Research and policy agenda
}

\author{
N J Ngcobo, ${ }^{1}$ MB ChB, MSc Med (Bioethics and Health Law), MBA, DOH, DTM\&H, DCH; R J Burnett, ${ }^{2} \mathrm{MPH}, \mathrm{PhD} ; \mathrm{S}$ Cooper, ${ }^{3} \mathrm{MPH}, \mathrm{PhD}$; \\ C S Wiysonge, ${ }^{4} \mathrm{MD}, \mathrm{MPhil}, \mathrm{PhD}$ \\ ${ }^{1}$ Institute for Leadership and Research, Pretoria, South Africa \\ ${ }^{2}$ South African Vaccination and Immunisation Centre, Department of Virology, School of Medicine, Sefako Makgatho Health Sciences University, \\ Pretoria, South Africa \\ ${ }^{3}$ Cochrane South Africa, South African Medical Research Council, Cape Town, South Africa \\ ${ }^{4}$ Cochrane South Africa, South African Medical Research Council; and School of Public Health and Family Medicine, Faculty of Health Sciences, \\ University of Cape Town, South Africa
}

Corresponding author: N J Ngcobo (ntombenhle1m@gmail.com)

\begin{abstract}
Cervical cancer is responsible for one-quarter of a million deaths per year worldwide. In South Africa (SA), cervical cancer is the leading cause of cancer deaths among women aged 15 - 44 years. Human papillomavirus (HPV) vaccines provide a safe and highly effective means to reduce the burden of cervical cancer. The World Health Organization initiated a plan for the elimination of cervical cancer; the programme's success relies on the introduction and high uptake of HPV vaccines globally. SA introduced a school-based HPV vaccination programme in 2014, but uptake is not as high as expected. Suboptimal HPV vaccination coverage may result from various factors, including vaccine hesitancy. Vaccine-hesitant parents may delay or refuse HPV vaccination for their daughters. Tailored interventions are needed to address this. However, knowledge regarding vaccine hesitancy and policies to address this hesitancy in SA are currently limited. While SA has taken commendable steps in cervical cancer prevention by implementing and financing the HPV vaccination programme, it is imperative that there are clear policies in place to help strengthen the programme. These policies need to clarify areas of uncertainty that may lead to mistrust, and pre-empt factors that will cause hesitancy. Equally important is that local research should be conducted to better understand HPV vaccination hesitancy and other determinants of uptake to further inform and shape national policies.
\end{abstract}

S Afr Med J 2019;109(1):13-15. DOI:10.7196/SAMJ.2019.v109i1.13723

Cervical cancer is a public health scourge that affects more than half a million women globally, more than half of whom die of the disease every year. ${ }^{[1]}$ Low- and middle-income countries (LMICs), especially those in sub-Saharan Africa (SSA), bear the greatest burden of cervical cancer. Malawi, Mozambique and Comoros have the highest incidence of the disease in the world $(75.9,65.0$ and 61.3 per 100000 women, respectively). This is in contrast to European countries, such as Germany, France and Switzerland, which have an incidence of 8.2, 6.8 and 3.6 per 100000 women, respectively. SSA is home to $10.5 \%$ of the global population of women aged $\geq 15$ years; yet, the region accounts for $21.6 \%$ and $17.7 \%$ of the global burden of cervical cancer deaths and cases, respectively. ${ }^{[1]}$

In South Africa (SA), cervical cancer is the second most common cancer that occurs in women and the leading cause of cancer deaths among women aged 15 - 44 years, with an estimated annual incidence of 7735 cases and 4248 deaths a year. ${ }^{[2]}$ Given the high incidence of HIV infection, which predisposes women to cervical cancer, and the low uptake of cervical cancer screening, the incidence of and mortality from cervical cancer in SSA and SA can be expected to rise in the absence of primary prevention. ${ }^{[3]}$

Infection with high-risk types of human papillomavirus (HPV) is the cause of cervical cancer, which can be prevented by HPV vaccination. ${ }^{[1]}$ The latter has been demonstrated to be safe and highly effective, providing up to $98.2 \%$ protection against specific high-risk strains of $\mathrm{HPV}^{[4-6]} \mathrm{HPV}$ vaccines have been available and used in several LMICs and high-income countries (HICs) for more than a decade. In SA, the bivalent and quadrivalent HPV vaccines have been available in the private sector since 2008. In 2014, a schoolbased HPV vaccination programme was introduced, with 2 doses (6 months apart, administered during 1-month campaigns) of the bivalent HPV vaccine offered free of charge to grade 4 girls aged $\geq 9$ years in public sector schools. ${ }^{[7]}$

\section{Human papillomavirus vaccination} uptake and hesitancy

Uptake of the HPV vaccine through the school-based programme in SA has been reported to be fairly good, with $85 \%$ coverage for the first dose in 2014 that targeted about 500000 female learners. ${ }^{[7,8]}$ Subsequent performance has been reported as numbers only and has not been converted to percentages. The reported number of vaccinated learners for 2014 - 2016 indicates a significant decrease between the first and second doses, which amounts to a decrease of $21.4 \%$ in 2014 and $26.0 \%$ in 2016 . Furthermore, the study commissioned by the National Department of Health $(\mathrm{NDoH})$ to assess the first round of the 2014 campaign reported pockets of low HPV vaccination coverage, which in two sub-districts were only $40 \%$ and $43 \% .{ }^{[7]}$

Suboptimal uptake of HPV vaccination is not unique to areas in SA, but remains a serious challenge worldwide. For example, a recent meta-analysis of data from 79 studies in 15 countries, including $>840000$ parents, found the overall parental uptake of $\geq 1$ doses of HPV vaccines for their children to be $41.5 \%{ }^{[9]}$ In 2016 in the USA, 
the $\geq 1$-dose HPV vaccination coverage among teens was $60.4 \%$ (65.1\% for females; $56.0 \%$ for males), which was an improvement on previous years. ${ }^{[10]}$ Coverage rates are also reported to vary greatly by region. ${ }^{[11]}$

Reasons for poor uptake of HPV vaccination are multifactorial. Supply-related factors, such as cost and unavailability of the vaccines; inadequate financing mechanisms; poor health system capabilities for vaccination; vaccine storage and cold-chain constraints; poor access to healthcare; limited and missed vaccination opportunities; and low prioritisation of adolescent health, are important contributors to suboptimal HPV vaccination uptake, particularly in SSA. ${ }^{[12-14]}$ However, vaccine hesitancy may be an important additional factor in the low uptake of HPV vaccination for school-based programmes that are designed to eliminate most of these obstacles.

According to the World Health Organization's Strategic Advisory Group of Experts on Immunization (SAGE) Working Group on Vaccine Hesitancy, vaccine hesitancy refers to: 'delays in acceptance or refusal of vaccination despite availability of vaccination services.' ${ }^{\text {'15] }}$

Vaccine hesitancy is known to vary across time, place and vaccines, and is believed to be influenced by confidence, complacency, convenience, risk calculation and collective responsibility. ${ }^{[15,16]}$ Vaccine-hesitant individuals range from those who may accept vaccination even when not fully convinced, to those who refuse vaccination because they have doubts regarding the necessity or safety of vaccines. ${ }^{[15]}$

The phenomenon of vaccine hesitancy in relation to all vaccination programmes, and specifically to HPV vaccination, has been described and studied extensively in HICs. Evidence from these countries suggests that hesitancy might be higher than for other childhood vaccines. ${ }^{[17,18]}$ These studies have identified the following as key issues underpinning HPV vaccination hesitancy: trust and safety concerns; lack of knowledge regarding the disease and vaccines among the targeted population, parents and healthcare workers (HCWs); and influence of peers and the community. ${ }^{[18-21]}$ Moreover, research has shown that HCW' recommendations are critical for HPV vaccination acceptance and uptake. For example, a US study found that, despite clear guidelines, many HCWs did not routinely recommend HPV vaccination. ${ }^{[22]}$ Importantly, studies have reported that some HCWs are hesitant and fail to make the recommendation at a critical time, when parents need encouragement, clarity and open discussion. ${ }^{[20,22]}$

It is, however, unclear whether these findings can be generalised to SA and other SSA countries, given that vaccine hesitancy is thought to be highly variable and context specific. ${ }^{[9]}$ Research on HPV vaccine hesitancy in SA is limited. Prior to the introduction of the school-based HPV vaccination programme in 2014, various studies explored knowledge, attitudes and beliefs regarding HPV and cervical cancer, as well as knowledge and acceptance of the HPV vaccine. ${ }^{[23-26]}$ A consistent finding across these studies was that knowledge and understanding of cervical cancer, the relationship between HPV and cervical cancer, and the purpose of HPV vaccination were low. The studies also revealed that parents might have various concerns regarding the HPV vaccine, including its safety and efficacy and its short- and long-term side-effects, and that HPV vaccination may encourage risky adolescent sexual behaviour. Moreover, the Vaccine and Cervical Cancer Screen (VACCS) project, a pilot school-based vaccination programme conducted in 19 primary schools in the Western Cape and Gauteng provinces prior to the national programme roll-out, indicated that suboptimal coverage was predominantly due to lack of parental consent. ${ }^{[27,28]}$ The project found that vaccine uptake among girls whose caregivers attended information evenings, which included addressing safety concerns, was significantly higher $(\sim 90 \%)$ than among girls whose caregivers did not attend $(\sim 50 \%)$. Parental concerns about safety may be linked to negative social media coverage of the HPV vaccine. This notion is supported by the $\mathrm{NDoH}$-commissioned post-introduction study, where some key informants reported that HCWs were 'nervous' about using the HPV vaccine, and that parental consent was negatively affected in areas where negative sentiments about the vaccine were shared on social media. ${ }^{[7]}$ Furthermore, anecdotal reports indicated refusal by some parents to give consent, including messages such as 'Do not touch my child' on the consent form (National EPI Manager personal communication, 30 March 2015).

Being a school-based programme, issues relating to communication and social mobilisation for the HPV vaccination programme should include various stakeholders, including the Department of Basic Education (DoE), specifically the educators, school governing bodies and parent associations. These and other community-based organisations should be sensitised to and informed on HPV as the cause of cervical cancer, and the benefits of HPV vaccination. DelanyMoretlwe et al. ${ }^{[7]}$ recommended that the DoE should play a more prominent role in communication and social mobilisation to increase demand for HPV vaccination. This is an important recommendation, considering that the DoE has ongoing contact and collaboration with these stakeholders, while the $\mathrm{NDoH}$ does not. It may go a long way towards strengthening the role of educators in promoting the vaccine, and ameliorate the potential threat posed by educators who may be hesitant to recommend and advocate HPV vaccination.

\section{Research agenda and policy issues}

The limited research on and context-specific issues of the HPV vaccination programme in SA point to a need for increased research on the potential existence, nature and causes of vaccine hesitancy in this programme. The setting and circumstances are different to many of those in HICs, and therefore unique challenges and concerns might exist. For example, the programme does not include private schools and is not available at public healthcare facilities. This may be a source of concern and undermine trust, as some may question the NDoH's motive for HPV vaccination in public schools. Furthermore, being grade based, the programme excludes a large number of pre-adolescent, adolescent and young women who are eligible for and would benefit from the vaccine. This includes a large population of learners attending public and private schools. Moreover, there is no readily available information on the uptake of the HPV vaccine in the private sector. These issues, and their possible negative impact on the prevalence of vaccine hesitancy, need to be further researched in SA.

More specifically, research areas that need to be addressed in SA, and potentially other LMICs, are the following:

- Extent of HPV vaccine hesitancy in the public sector among parents of targeted girls and those eligible for the national schoolbased HPV vaccination programme.

- Reasons behind the high drop-out rate between the first and second doses of HPV vaccine in this programme.

- Knowledge, beliefs, attitudes and practices of girls and boys (in the general population) who are eligible for the HPV vaccine but are not targeted by the national programme.

- HPV vaccination coverage, HPV vaccination hesitancy, reasons for non-vaccination, and sociodemographic factors related to HPV vaccination status in the private sector.

- The role of public and private HCWs in HPV vaccination uptake and the likelihood of HCWs recommending HPV vaccination to 
the eligible population, especially considering that a significant proportion of this population is not targeted by the national programme, and many learners in private schools have medical insurance and can afford the vaccine.

- The role of educators in promoting HPV vaccination uptake, and the existence and extent of HPV vaccine hesitancy among educators.

- The role played by social media in influencing HPV vaccination uptake.

- Obtaining consent for HPV vaccination, and weighing the benefits and risks of the current approach to obtaining consent to alternative approaches used in other parts of the world.

Although the literature is based on research from HICs, it provides LMICs, such as SA, with enough background to develop policies on HPV vaccination that can be continuously shaped by evidence from local research. There are a number of policy issues that need to be addressed, including:

- A clear policy on HPV vaccination to address the issue of HPV vaccination in public and private sectors, clarifying why the vaccine is not provided in private schools.

- Communication and social mobilisation for HPV vaccination, which are not only focused on parents and girls targeted by the programme. Communication should be broad based and widely accessible, targeting the public at large, including all parents, young pre-adolescents and adolescents who are eligible for HPV vaccination. This focus should include learners (girls and boys) in private and public sector schools, who are in grade 5 and higher and have not received the HPV vaccine.

- The role of the DoE in communicating and advocating the HPV vaccination programme.

- Standard operating procedures for HCWs to ensure that there are no missed opportunities in recommending and providing the HPV vaccine. These HCWs include paediatricians, general practitioners and those in school, adolescent and youth health and primary healthcare clinics.

- An integrated approach to provision of the HPV vaccine with other services, e.g. youth and adolescent health, school health, reproductive health, primary healthcare and health promotion. Guidelines and policies of these programmes should be adapted to allow integration with HPV vaccination.

- Vaccinology training that results in accreditation of HCWs who interact with the targeted population, including training on HPV infection, HPV vaccines, cervical cancer and related matters. This will allow HCWs to confidently promote vaccines, including the HPV vaccine.

The extent and impact of vaccine hesitancy need to be investigated so that focused interventions may be implemented and appropriate policies developed and adopted. It is envisaged that addressing these research and policy gaps will help to improve uptake of the HPV vaccine, overcome HPV vaccination hesitancy and move SA closer to attaining the goal of the global vaccine action plan of creating a society that values immunisation as a social good and demands it as their right and responsibility. ${ }^{[29]}$

\section{Declaration. None.}

Acknowledgements. C S Wiysonge's work is supported by the South African Medical Research Council and the National Research Foundation of South Africa (grant no. 106035). R J Burnett's work is supported by the National Research Foundation of South Africa (grant no. 98959).
Author contributions. NJN: drafted the initial manuscript and the key subtopics, and continued update of the manuscript. RJB: reviewed and critiqued the initial version and introduced additional references. SC: updated the earlier draft, re-outlined the manuscript and added another aspect on hesitancy, as well as references. CSW: initiated the manuscript and introduced the specific focus on local experience of HPV vaccination hesitancy, with references.

Funding. None.

Conflicts of interest. None.

1. Bruni L, Barrionuevo-Rosas L, Albero S, et al. ICO/IARC Information Centre on HPV and Cancer Human papillomavirus and related diseases in the world. Summary report. 2017. http://www. hpvcentre.net/statistics/reports/XWX.pdf (accessed 2 October 2018).

2. Bruni L, Barrionuevo-Rosas L, Albero S, et al. ICO/IARC Information Centre on HPV and Cancer Human papillomavirus and related diseases in South Africa. Summary report. 2017. http://www. hpvcentre.net/statistics/reports/ZAF.pdf (accessed 2 October 2018).

3. Menon S, Luchters S, Rossi R, et al. Human papillomavirus correlates of high grade cervical dysplasia in HIV-infected women in Mombasa, Kenya: A cross sectional analysis. Virol J 2018;15(1):54. https:// doi.org/10.1186/s12985-018-0961-3

4. Kjaer SK, Sigurdsson K, Iversen OE, et al. A pooled analysis of continued prophylactic efficacy of quadrivalent human papillomavirus (types 6/11/16/18) vaccine against high-grade cervical and externa genital lesions. Cancer Prev Res 2009;2(10):868-878. https://doi.org/10.1158/1940-6207.CAPR-09-0031

5. Wheeler CM, Castellsague X, Garland SM, et al. Cross-protective efficacy of HPV-16/18 AS04adjuvanted vaccine against cervical infection and pre-cancer caused by non-vaccine oncogenic HPV types: 4-year end-of-study analysis of the randomised, double-blind PATRICIA trial. Lancet Onco 2012;13(1):100-110. https://doi.org/10.1016/S1470-2045(11)70287-X

6. Arbyn M, Xu L, Simoens C, Martin-Hirsch PPL. Prophylactic vaccination against human papillomaviruses to prevent cervical cancer and its precursors. Cochrane Database Syst Rev 2018;(5):CD009069. https://doi.org/10.1002/14651858.CD009069.pub3

7. Delany-Moretlwe S, Kelley KF, James S, et al. Human papillomavirus vaccine introduction in South Africa: Implementation lessons from an evaluation of the national school-based vaccination campaign. Glob Health Sci Pract 2018;6(3):425-438. https://doi.org/10.9745/GHSP-D-18-00090

8. Denny L, Kuhn L. Cervical cancer prevention and early detection from a South African perspective. In: Padarath A, Barron P, eds. South African Health Review 2017. Durban: Health Systems Trust, 2017 Newman PA, Logie CH, Lacombe-Duncan A, et al. Parents' uptake of human papillomavirus vaccines
for their children: A systematic review and meta-analysis of observational studies. BMJ Open for their children: A systematic review and meta-analysis of obst
2018;8(4):e19206. https://doi.org/10.1136/bmjopen-2017-019206

10. Centers for Disease Control and Prevention. National, regional, state, and selected local area vaccination Centers for Disease Control and Prevention. National, regional, state, and selected local area vaccination
coverage among adolescents aged 13 - 17 years - United States, 2016. MMWR 2017;66(33):874-882.

coverage among adolescents aged 13 - 17 years - United States, 2016. MMWR 2017;66(33):874-882.
11. Bruni L, Diaz M, Barrionuevo-Rosas L, et al. Global estimates of human papillomavirus vaccination Bruni L, Diaz M, Barrionuevo-Rosas L, et al. Global estimates of human papillomavirus vaccination
coverage by region and income level: A pooled analysis. Lancet Glob Health 2016;4(7):e453-463. coverage by region and income level: A pooled
https://doi.org/10.1016/S2214-109X(16)30099-7

12. Brotherton JM, Ogilvie GS. Current status of human papillomavirus vaccination. Curr Opin Oncol 2015;27(5):399-404. https://doi.org/10.1097/CCO.0000000000000211

13. Bloem P, Ogbuanu I. Vaccination to prevent human papillomavirus infections: From promise to practice. PLOS Med 2017;14(6):e1002325. https://doi.org/10.1371/journal.pmed.1002325

14. Gallagher KE, LaMontagne DS, Watson-Jones D. Status of HPV vaccine introduction and barriers to country uptake. Vaccine 2018;36(32):4761-4767. https://doi.org/10.1016/j.vaccine.2018.02.003

5. MacDonald NE, the SAGE Working Group on Vaccine Hesitancy. Vaccine 2015;33(34):4161-4164. https://doi.org/10.1016/j.vaccine.2015.04.036

16. Cooper S, Betsch C, Sambala EZ, et al. Vaccine hesitancy - a potential threat to the achievements of vaccination programmes in Africa. Hum Vaccin Immunother 2018;14(10):2355-2357. https://doi. org / 10.1080/21645515.2018.1460987

17. Jorgensen L, Gøtzsche PC, Jefferson T. The Cochrane HPV vaccine review was incomplete and ignored important evidence of bias. BMJ Evidence-Based Med 2018;23(5):165-168. https://doi.org/10.1136/ bmjebm-2018-111012

18. Gilkey M, Moss J, Coyne-Beasley T, Shah P, Brewer N. Physician communication about adolescent vaccination: How is human papillomavirus vaccine different? Prevent Med 2015;77:181-185. https:// doi.org/10.1016/j.ypmed.2015.05.024

19. Betsch C, Böhm R, Chapman GB. Using behavioral insights to increase vaccination policy effectiveness. Policy Insights Behav Brain Sci 2015;2(1):61-73. https://doi.org/10.1177/2372732215600716

20. Patel P, Berenson A. Sources of HPV vaccine hesitancy in parents. Hum Vaccines Immunother 2013;9(12):2649-2653. https://doi.org/10.4161/hv.26224

21. Fontenot H, Domush V. Parental attitudes and beliefs regarding the nine-valent human papillomavirus . Fontenot H, Domush V. Parental attitudes and beliefs regarding the nine-valent human papillom
vaccine. J Adolesc Health 2015;57(6):595-600. https://doi.org/10.1016/j.jadohealth.2015.09.003

22. Gaccine. J Adolesc Health 2015;57(6):595-600. https://doi.org/10.1016/j.jadohealth. 2015.09 .003
Gilkey McRee A-L. Provider communication about HPV vaccination: A systematic review. Hum Gilkey M, McRee A-L. Provider communication about HPV vaccination: A systematic review.
Vaccines Immunother 2016;12(6):1454-1468. https://doi.org/10.1080/21645515.2015.1129090

23. Harries J, Moodley J, Barone MA, et al. Preparing for HPV vaccination in South Africa: Key challenges Harries J, Moodley J, Barone MA, et al. Preparing for HPV vaccination in South Africa:
and opinions. Vaccine 2009;27(1):38-44. https://doi.org/10.1016/j.vaccine.2008.10.033

and opinions. Vaccine 2009;27(1):38-44. https://doi.org/10.1016/j.vaccine. 2008.10 .033
24. Katz IT, Nkala B, Dietrich J, et al. A qualitative analysis of factors influencing HPV vaccine uptake in Katz IT, Nkala B, Dietrich J, et al. A qualitative analysis of factors influencing HPV vaccine uptake in
Soweto, South Africa among adolescents and their caregivers. PLOS ONE 2013;8(8):e72094. https:// doi.org/10.1371/journal.pone.007209

25. Francis SA, Battle-Fisher M, Liverpool J, et al. A qualitative analysis of South African women's knowledge, attitudes, and beliefs about HPV and cervical cancer prevention, vaccine awareness and acceptance, and maternal-child communication about sexual health. Vaccine 2011;29(47):8760-8765. https://doi.org/10.1016/j.vaccine.2011.07.116

26. Francis SA, Nelson J, Liverpool J, et al. Examining attitudes and knowledge about HPV and cervical cancer risk among female clinic attendees in Johannesburg, South Africa. Vaccine 2010;28(50):8026-8032. https://doi.org/10.1016/j.vaccine.2010.08.090

27. Botha MH, van der Merwe FH, Snyman LC, et al. The vaccine and cervical cancer screen (VACCS project: Acceptance of human papillomavirus vaccination in a school-based programme in two provinces of South Africa. S Afr Med J 2015;105(1):40-43. https://doi.org/10.7196/SAMJ.841

28. Botha MH, Richter KL. Cervical cancer prevention in South Africa: HPV vaccination and screening both essential to achieve and maintain a reduction in incidence. S Afr Med J 2015;105(1):33-34. https:// doi.org/10.7196/SAMJ.9233

29. World Health Organization. Global Vaccine Action Plan 2011 - 2020. Geneva: WHO, 2013. 